CLINICAL STUDY

\title{
Measures of bioavailable serum testosterone and estradiol and their relationships with muscle mass, muscle strength and bone mineral density in postmenopausal women: a cross-sectional study
}

\author{
Tineke A C M van Geel, Piet P Geusens ${ }^{1}$, Bjorn Winkens ${ }^{2}$, Jean-Pierre J E Sels ${ }^{1}$ and Geert-Jan Dinant \\ Department of General Practice, Maastricht University, PO Box 616, 6200 MD Maastricht, The Netherlands, ${ }^{1}$ Department of Internal Medicine, \\ Maastricht University Medical Centre, PO Box 5800, 6202 AZ Maastricht, The Netherlands and ${ }^{2}$ Department of Methodology and Statistics, Maastricht \\ University, PO Box 616, 6200 MD Maastricht, The Netherlands \\ (Correspondence should be addressed to T A C M van Geel; Email: t.vangeel@hag.unimaas.nl)
}

\begin{abstract}
Objective: The physiologic role of circulating endogenous testosterone and estrogen concentrations in relation to lean body mass (LBM) and muscle strength is not as well documented in postmenopausal women as in elderly men.

Design: Three hundred and twenty-nine healthy postmenopausal women were randomly selected from a general practice population-based sample aged between 55 and 85 years.

Methods: Total testosterone and estrogen (TT and TE) and sex hormone-binding globulin (SHBG) were determined and estimates of bioavailable testosterone (free androgen index (TT/SHBG, FAI), calculated free testosterone (cFT), and estrogen (TE/SHBG, ESR) were calculated. Examinations included bone mineral density (BMD) of the spine and femoral neck (FN), LBM, maximum quadriceps extension strength (MES) and maximum handgrip strength (MGS), timed up-and-go test (TUGT), osteocalcin (OC), and urinary deoxy-pyridinoline/creatinine (DPyr). Correlations were assessed using Pearson's correlation coefficient $(r)$.

Results: With advancing age, LBM, MES, MGS, BMD, and ESR significantly declined (range $r$ : -0.356 to -0.141 ) and TUGT, and DPyr significantly increased (range $r: 0.135$ to $0.282(P<0.05)$ ). After age-adjustment, LBM, MES, and BMD in spine and FN were significantly related to bioavailable testosterone (range $r$ : 0.146 to 0.193 , for cFT, and 0.157 to 0.224 , for FAI) and to ESR (range $r: 0.162$ to 0.273$)$. OC and DPyr were significantly inversely related to ESR $(r$ : -0.154 and -0.144 respectively).

Conclusions: Age-related loss of LBM, MES and BMD in postmenopausal women is partly dependent on the presence of endogenous bioavailable testosterone and estrogen.
\end{abstract}

European Journal of Endocrinology 160 681-687

\section{Introduction}

It is well documented that part of the bone loss that occurs with ageing in postmenopausal women and elderly men is related to a decrease in serum levels of bioavailable estrogen and testosterone (1-5), and that drugs with estrogenic and/or androgenic properties can be used to prevent age-related bone loss $(1,6,7)$.

It has been shown that part of the loss of lean (fatfree) body mass, muscle mass, and maximum voluntary strength that occurs with ageing in elderly men is related to bioavailable testosterone $(1,8)$, and most, though not all, studies have found that testosterone administration to hypogonadal and eugonadal men is associated with dose- and concentration-dependent increase in lean mass, muscle mass, and maximum voluntary strength $(7-10)$. In men, testosterone increases muscle protein synthesis and satellite cell number, probably by stimulating the differentiation of mesenchymal pluripotent cells to the myogenic lineage (10), resulting in muscle fiber hypertrophy and myonuclear number increase $(10,11)$.

In postmenopausal women, not enough is known about the relation between serum estrogen and the decrease in muscle mass and muscle strength, and the physiological significance of low testosterone in relation to muscle mass and muscle strength remains unclear $(12,13)$. Data on the effects of estrogen and testosterone administration on muscle mass and muscle strength in postmenopausal women are scarce, much more so than those for elderly men $(14,15)$.

Serum testosterone and estrogen are mainly bound to sex-hormone binding globulin (SHBG) and albumin. Albumin-bound and free testosterone and estrogen are 
believed to have access to target tissues $(1,16)$. Several methods to calculate free testosterone from adequately measured testosterone and SHBG by immunoassay are available and some have been shown to be a reliable index of bioavailable testosterone (17). The same holds for estrogen (1).

Therefore, the main analyses in this cross-sectional study of 329 postmenopausal women, focused on the hypothesis that the decreases in muscle mass and muscle strength with age are related to the drop in circulating endogenous testosterone and estrogen concentrations.

\section{Materials and methods}

\section{Subjects}

Twenty general practitioners from 10 general practice centers participated in the original population-based cohort study. The region consists of two towns in the southern part of the Netherlands, surrounded by suburban villages (18). We drew a random sample of 370 postmenopausal women aged between 55 and 85 years from the 4203 women who were included in the original study. All participating women signed an informed consent form.

\section{Muscle mass and muscle strength}

The assessments started in 1998-1999 with weight (in $\mathrm{kg}$ ), height (in $\mathrm{cm}$ ) and total body lean and fat mass (including head, LBM and FBM), measured by dualenergy X-ray absorptiometry (DXA; Hologic QDR-1000, Hologic Europe, Brussels, Belgium).

Quadriceps extension strength was measured using the Chatillon DFIS-200 (Largo, FL, USA), and handgrip strength using the Jamar dynamometer (Sammons Preston, Jackson, MI, USA). Quadriceps muscle strength was measured thrice in both legs and the handgrip strength thrice in both hands. Maximum quadriceps extension (MES) and handgrip strength (MGS) were defined as the maximum strength of the left or right leg/hand, whichever was the largest.

Mobility was tested using the timed up-and-go test (TUGT)(19) that measures the time needed to get up from a chair, walk three meters, turn, walk back, and sit down again.

\section{Hormone measurements}

Blood samples were collected in the morning after an overnight fast and first morning urine samples were collected as well. All laboratory tests, except for urinary creatinine (performed measured with the methods used in routine practice), were obtained with the IMMULITE device (Diagnostic Products Corporation (DPC), Los Angeles, CA, USA).
RIA was used to measure SHBG, intra-coefficient of variation $(\mathrm{CV}): 2.4-4.4 \%$ and inter-CV: $3.7-7.0 \%$ ), testosterone (intra-CV of 7.1 to $13.0 \%$ and inter-CV of 7.7 to $16.4 \%$ ) and $\mathrm{E}_{2}$ (E, intra-CV of 4.3 to $9.9 \%$ and inter-CV of 6.7 to $16.0 \%)(20)$.

Based on these values, parameters of bioavailable testosterone and estrogen were calculated according to the methods proposed in the endocrine literature (Table 1). These included the free androgen index (FAI) and calculated free testosterone (cFT). cFT was calculated using the value of 'binding of testosterone to SHBG' constant by Vermeulen et al. (KTS, Table 1) (17).

Bone turnover markers (BTM) including osteocalcin (OC, intra-CV of $2.2-4.0 \%$ and inter-CV of $5.1-9.9 \%$ ) and deoxypyridinoline/creatinine ratio (DPyr, intra-CV of $3.1-10.0 \%$ and inter-CV of $4.3-12.0 \%$ ) were measured using RIA.

\section{Bone mineral density}

Bone mineral density (BMD) was measured at the lumbar spine and at the femoral neck $(\mathrm{FN})$ by the same DXA instrument as used for LBM and FBM.

The quality control is performed in cooperation with the supplier of the DXA devices (Hologic). In addition, daily quality control was performed by the Department of Nuclear Medicine/Radiology. All measurements were performed in one center using the same device.

\section{Statistical analysis}

We based our analysis on methods similar to those used by Van den Beld et al. (1).

Table 1 Measurements and calculations for the various concentrations of testosterone and estrogen.

\begin{tabular}{|c|c|}
\hline & Measurements and calculations \\
\hline SHBG (nmol/l) & RIA (DPC, IMMULITE2000, Los Angeles, CA) \\
\hline TT (nmol/l) & RIA (DPC, IMMULITE2000, Los Angeles, CA) \\
\hline $\mathrm{FAI}(\% \mathrm{M} / \mathrm{M})$ & $\mathrm{FAI}=100 * \mathrm{TT} / \mathrm{SHBG}$, with both in $\mathrm{nmol} / \mathrm{l}$ \\
\hline \multirow[t]{4}{*}{$\mathrm{cFT}(\mathrm{pmol} / \mathrm{l})$} & According to Vermeulen et al. (17): \\
\hline & $\mathrm{cFT}=\left(-b+\sqrt{ }\left(b^{2}+4 a(\mathrm{TT})\right) / 2 a\right.$ \\
\hline & $\begin{array}{l}a=\mathrm{KTA}+\mathrm{KTS}+(\mathrm{KTA} \times \mathrm{KTS})((\mathrm{SHBG})+ \\
\quad(\text { albumin })-(\mathrm{TT}))\end{array}$ \\
\hline & $\begin{aligned} b=1+\mathrm{KTS}(\mathrm{SHBG})+\mathrm{KTA} \text { (albumin) } & \\
& -(\mathrm{KTA}+\mathrm{KTS})(\mathrm{TT})\end{aligned}$ \\
\hline FTR $(\% \mathrm{M} / \mathrm{M})$ & $\begin{array}{l}\mathrm{FTR}=(\mathrm{cFT}) /(\mathrm{TT}) \text {, with } \mathrm{cFT} \text { in } \mathrm{pmol} / \mathrm{l}, \mathrm{TT} \text { in } \\
\mathrm{nmol} / \mathrm{l}\end{array}$ \\
\hline Estradiol (pmol/l) & RIA (DPC, IMMULITE2000, Los Angeles, CA) \\
\hline ESR (pM/nM) & $\begin{array}{l}E S R=\left(E_{2}\right) /(S H B G) \text {, with } E_{2} \text { in } \mathrm{pmol} / \mathrm{l} \\
S H B G \text { in } \mathrm{nmol} / /\end{array}$ \\
\hline Osteocalcin (mg/l) & RIA (DPC, IMMULITE2000, Los Angeles, CA) \\
\hline Dpyr (nM/mM) & $\begin{array}{l}\text { Ppyr }=(P Y R) /(C R E) \text {, with PYR in } \mathrm{nmol} / \mathrm{l}, \mathrm{CRE} \\
\text { in } \mathrm{mmol} / \mathrm{l}\end{array}$ \\
\hline
\end{tabular}

SHBG, sex-hormone binding globulin; TT, total testosterone; FAI, free androgen index =total testosterone/SHBG; cFT, calculated free testosterone; FTR, free testosterone/total testosterone ratio; KTS, value of the 'binding of testosterone to SHBG' constant ( $\mathrm{l} / \mathrm{mol}), 10.0^{*}$ t8, per (Vermeulen); $\mathrm{KTA}$, value of the 'binding of testosterone to albumin' constant $(\mathrm{I} / \mathrm{mol}), 3.60$ * $t 4$, per (Vermeulen); $t 4=10^{4}$; (17) $E S R=E_{2} / S H B G$ ratio; DPyr, deoxypyridinoline/creatinine ratio. 
The relation between muscle strength, biochemical markers, and BMD with age was assessed using Pearson's correlation coefficient $(r)$. The $r$ shows the number of S.D. that the outcome will change as a result of one s.D. change in age (21). The differences between the highest and the lowest age-groups were calculated using the variables which significantly correlated with age. Partial correlation coefficients (partial $r$ ) were analyzed to assess the age-adjusted relation between LBM, muscle strength parameters, and biochemical markers. The age- and body mass index (BMI)-adjusted relation between these variables was also calculated.

SPSS software (version 15.0; SPSS Inc., Chicago, IL, USA) was used for the statistical analysis.

\section{Ethics}

The ethics review committee of the Maastricht University and the Maastricht University Hospital approved the study (reference number MEC 94-196.1).

\section{Results}

Of the 352 women who agreed to participate (response rate $=95.1 \%$ ), nine women were excluded from further analyses due to severe mobility problems (e.g., wheelchair dependent, leg prosthesis, Parkinson's disease, amyotrophic lateral sclerosis). Biochemical markers were available for 329 of the remaining 343 women $(95.9 \%)$.

\section{Changes in testosterone, estrogen, muscle mass, muscle strength, and BTM with age}

Table 2 presents the descriptive data. LBM, MES, and MGS declined with ageing, while the TUGT significantly increased with increasing age (Table 3). Women in the lowest age-group ( $<60$ years, $n=49,14.9 \%$ of the total) and in the highest age-group ( $>75$ years, $n=45,13.7 \%$ of the total) were compared in terms of the significant variables. LBM, MES and MGS declined by $4.9 \% \quad(P=0.049), 29.9$ and $24.3 \% \quad(P<0.001)$ respectively, while the TUGT increased by $39.0 \%$ $(P<0.001)$.

The descriptive data of the hormone concentrations and BTM are presented in Table 4, while Table 5 presents the relation between hormones and BTM and age. The IMMULITE is suitable for measurements at low concentration levels expected for postmenopausal women (20). The intra-assay precision profile (intra CV) for estradiol at a mean of $23.1 \mathrm{pg} / \mathrm{ml}(84.9 / 3.671)$ and total testosterone (TT) at a mean of $43.3 \mathrm{ng} / \mathrm{dl}$ $(1.5 / 0.03467)$ are $\sim 11.5$ and $10.8 \%$ respectively $(22,23)$.

ESR significantly decreased with ageing, while DPyr significantly increased with ageing. Again, women in the lowest age-group were compared with the highest age-group in terms of the significant variables. ESR declined by $22.4 \%$, while DPyr increased by $16.7 \%$.

BMI was not significantly related to either MES (partial $r$ : 0.102, $P=0.08$ ) or MGS (partial $r$ : -0.044 , $P=0.423)$, but was significantly related to the TUGT (partial $r=0.154, P<0.01$ ). After age-adjustment TT

Table 2 Descriptive data of the study population $(n=329)$.

\begin{tabular}{|c|c|c|c|}
\hline & Mean & S.D. & Interquartile range \\
\hline BMD lumbar spine $\left(\mathrm{g} / \mathrm{cm}^{2}\right)$ & 0.937 & 0.170 & $0.817-1.035$ \\
\hline BMD lumbar spine ( $T$-score) & -1.29 & 1.54 & $-2.40--0.40$ \\
\hline BMD femoral neck $\left(\mathrm{g} / \mathrm{cm}^{2}\right)$ & 0.703 & 0.119 & $0.622-0.770$ \\
\hline BMD femoral neck ( $T$-score) & -1.92 & 1.19 & $-2.70--1.23$ \\
\hline Age (years) & 66.9 & 6.51 & 61.4-71.5 \\
\hline Postmenopausal years & 20.1 & 8.09 & $14.0-26.0$ \\
\hline BMI $\left(\mathrm{kg} / \mathrm{m}^{2}\right)$ & 27.6 & 4.57 & $24.7-29.7$ \\
\hline LBM $(\mathrm{kg})$ & 41.9 & 4.90 & $38.4-44.9$ \\
\hline Left arm (kg) & 2.09 & 0.35 & $1.85-2.29$ \\
\hline Right arm (kg) & 2.20 & 0.35 & $1.94-2.39$ \\
\hline Trunk $(\mathrm{kg})$ & 21.3 & 2.77 & $19.4-22.8$ \\
\hline Left leg (kg) & 6.48 & 0.90 & $5.81-7.12$ \\
\hline Right leg (kg) & 6.73 & 0.97 & $6.01-7.42$ \\
\hline Head $(\mathrm{kg})$ & 3.02 & 0.28 & $2.80-3.23$ \\
\hline FBM $(\mathrm{kg})$ & 25.9 & 6.93 & $21.7-30.1$ \\
\hline Left arm (kg) & 1.78 & 0.69 & $1.34-2.04$ \\
\hline Right arm (kg) & 1.88 & 0.71 & $1.39-2.20$ \\
\hline Trunk $(\mathrm{kg})$ & 12.6 & 4.16 & $10.2-14.8$ \\
\hline Left leg (kg) & 4.27 & 1.26 & $3.57-5.20$ \\
\hline Right leg (kg) & 4.45 & 1.26 & $3.57-5.20$ \\
\hline Head $(\mathrm{kg})$ & 0.90 & 0.10 & $0.83-0.97$ \\
\hline MES (kg) & 23.4 & 7.77 & $17.8-28.0$ \\
\hline MGS (kg) & 26.4 & 5.90 & $22.0-30.0$ \\
\hline TUGT (s) & 13.4 & 7.78 & $9.90-14.6$ \\
\hline
\end{tabular}

S.D., standard deviation; BMD, bone mineral density; LBM, lean body mass; FBM, fat body mass; MES; maximum extension quadriceps strength; MGS, maximum handgrip strength; TUGT, timed up-and-go test. 
Table 3 Relationship between physical characteristics and age $(n=329)$.

\begin{tabular}{|c|c|c|}
\hline & \multicolumn{2}{|c|}{ Age (years) } \\
\hline & $r$ & $P$ value \\
\hline BMD lumbar spine $\left(\mathrm{g} / \mathrm{cm}^{2}\right)$ & -0.152 & 0.006 \\
\hline BMD lumbar spine ( $T$-score) & -0.149 & 0.007 \\
\hline BMD femoral neck (FN, $\left.\mathrm{g} / \mathrm{cm}^{2}\right)$ & -0.290 & $<0.001$ \\
\hline BMD femoral neck ( $T$-score) & -0.300 & $<0.001$ \\
\hline BMI $\left(\mathrm{kg} / \mathrm{m}^{2}\right)$ & -0.053 & 0.348 \\
\hline LBM $(\mathrm{kg})$ & -0.166 & 0.003 \\
\hline Left arm (kg) & -0.150 & 0.008 \\
\hline Right arm (kg) & -0.149 & 0.009 \\
\hline Trunk $(\mathrm{kg})$ & -0.149 & 0.008 \\
\hline Left leg (kg) & -0.138 & 0.015 \\
\hline Right leg (kg) & -0.127 & 0.025 \\
\hline Head $(\mathrm{kg})$ & -0.022 & 0.700 \\
\hline FBM $(\mathrm{kg})$ & 0.033 & 0.557 \\
\hline Left arm (kg) & 0.032 & 0.579 \\
\hline Right arm (kg) & 0.039 & 0.490 \\
\hline Trunk $(\mathrm{kg})$ & 0.077 & 0.173 \\
\hline Left leg (kg) & -0.040 & 0.487 \\
\hline Right leg (kg) & -0.028 & 0.628 \\
\hline Head $(\mathrm{kg})$ & 0.018 & 0.757 \\
\hline MES (kg) & -0.318 & $<0.001$ \\
\hline MGS $(\mathrm{kg})$ & -0.356 & $<0.001$ \\
\hline TUGT (s) & 0.282 & $<0.001$ \\
\hline
\end{tabular}

(partial $r=0.075, P=0.178$ ) was not significantly related to BMI, but the FAI (partial $r=0.215$, $P<0.001)$ and $c F T$ were significantly related to BMI (partial $r=0.3274, P<0.001$ ).

SHBG and FAI or cFT (partial $r$ : -0.440 or -0.348 respectively) were significantly negatively related, while the FAI and cFT or TT (partial $r$ : 0.947 or 0.672 respectively) were positively related $(P<0.001)$. The relations between estradiol and SHBG (partial $r$ : 0.187), ESR and SHBG, FAI or cFT (partial $r$ : $-0.556,0.532$ or 0.330 respectively) were also significant $(P<0.001)$.

Table 4 Summed values of hormone levels in serum $(n=329)$.

\begin{tabular}{llll}
\hline & Mean & s.D. & Interquartile range \\
\hline SHBG $(\mathrm{nmol} / \mathrm{l})$ & 53.3 & 30.2 & $34.4-63.0$ \\
TT $(\mathrm{nmol} / \mathrm{l})$ & 1.50 & 0.959 & $0.689-2.00$ \\
FAI $(\% \mathrm{M} / \mathrm{M})$ & 3.66 & 3.41 & $1.54-4.53$ \\
cFT (pmol/l) & 22.4 & 17.4 & $10.7-28.2$ \\
FTR $(\% \mathrm{M} / \mathrm{M})$ & 1.49 & 0.487 & $1.17-1.76$ \\
Estradiol $\left(\mathrm{E}_{2}, \mathrm{pmol} / \mathrm{l}\right)$ & 84.9 & 47.5 & $73.4-73.4$ \\
E $_{2} / \mathrm{SHBG}$ ratio $(\mathrm{ESR}$, & 2.06 & 1.49 & $1.22-2.26$ \\
$\quad$ pM/nM) & & & \\
Osteocalcin (mg/l) & 3.47 & 2.32 & $1.68-4.70$ \\
DPyr (nM/mM) & 6.29 & 2.34 & $4.87-7.24$ \\
\hline
\end{tabular}

S.D., standard deviation. FAI, TT/SHBG; FTR, cFT10/TT; DPyr, Pyrilinks$\mathrm{D} /$ creatinine; SHBG, sex-hormone binding globulin; TT, total testosterone; FAl, free androgen index; cFT, calculated free testosterone; FTR, free testosterone/total testosterone ratio; DPyr, deoxypyridinoline/creatinine ratio.
Table 5 Relationship between age and hormone levels $(n=329)$.

\begin{tabular}{lcr}
\hline & \multicolumn{2}{c}{ Age (years) } \\
\cline { 2 - 3 } & $r$ & $P$ value \\
\hline SHBG $(\mathrm{nmol} / \mathrm{l})$ & 0.091 & 0.102 \\
TT $(\mathrm{nmol} / \mathrm{l})$ & 0.034 & 0.540 \\
FAI $(\% \mathrm{M} / \mathrm{M})$ & -0.080 & 0.153 \\
cFT $(\mathrm{pmol} / \mathrm{l})$ & -0.043 & 0.438 \\
FTR & -0.142 & 0.011 \\
Estradiol $\left(\mathrm{E}_{2}, \mathrm{pmol} / \mathrm{l}\right)$ & -0.007 & 0.901 \\
E $_{2} / \mathrm{SHBG}$ ratio $(\mathrm{ESR}$, & -0.141 & 0.011 \\
pM/nM) & & \\
Osteocalcin (mg/l) & 0.050 & 0.372 \\
DPyr (nM/mM) & 0.135 & 0.015 \\
\hline
\end{tabular}

$r$, Pearson's correlation coefficient; SHBG, sex-hormone binding globulin; TT, total testosterone; FAl, free androgen index; CFT, calculated free testosterone; FTR, free testosterone/total testosterone ratio; DPyr, deoxypyridinoline/creatinine ratio.

As regards to testosterone, higher values of FAI and cFT were significantly positively related with LBM, FBM, and MES, after age-adjustment (Table 6).

As regards to estrogen, only higher values of ESR significantly increased LBM and FBM; it was not significantly related to the other muscle strength parameters (Table 6).

In addition, higher values of the BTM osteocalcin significantly increased LBM, (partial $r=-0.117$ $(P<0.05))$, while higher values of DPyr significantly decreased MGS (partial $r=-0.149(P<0.01)$ ) and significantly increased the TUGT (partial $r=0.126$ $(P<0.05))$.

\section{The relation of testosterone, estrogen, and BTM with BMD}

After adjustment for age, higher values of FAI, cFT, estradiol, and ESR were significantly positively related with $\mathrm{BMD}$ measured at the lumbar spine and at the $\mathrm{FN}$ (Table 6).

Of the BTM, higher values of OC significantly declined BMD measured at the lumbar spine (partial $r=-0.135$ $(P=0.016))$ and at the $\mathrm{FN}$ (partial $r=-0.171$ $(P=0.003))$.

\section{Discussion}

The results show that age-related loss of LBM, MES, and BMD is partly dependent on the presence of endogenous bioavailable testosterone and estrogen concentrations.

In contrast to the well-documented relations of estrogen and testosterone with BMD in women and men and with muscle strength and mass in men, not much attention has been given to the relations of testosterone and estrogen with muscle mass and muscle strength in postmenopausal women $(12,14)$. A study involving 20 women aged between 43 and 73 years old found a significant relation between free testosterone 
Table 6 Age-adjusted relations between hormone levels and physical characteristics.

\begin{tabular}{lccrrrrr}
\hline & \multicolumn{7}{c}{ Partial $r$} \\
\cline { 2 - 7 } & LBM & FBM & MES & MGS & TUGT & BMD L2-L4 & BMD FN \\
\hline SHBG (nmol/l) & $-0.282^{*}$ & $-0.210^{*}$ & -0.083 & -0.064 & -0.066 & -0.094 & $-0.129^{\ddagger}$ \\
TT (nmol/l) & 0.016 & 0.011 & 0.104 & -0.045 & -0.051 & 0.101 & 0.047 \\
FAI (\% M/M) & $0.224^{*}$ & $0.148^{\dagger}$ & $0.193^{*}$ & -0.021 & 0.005 & $0.157^{\dagger}$ & $0.138^{\ddagger}$ \\
cFT (pmol/l) & $0.167^{\dagger}$ & $0.190^{*}$ & $0.178^{\dagger}$ & -0.017 & -0.013 & $0.146^{\dagger}$ & $0.193^{*}$ \\
FTR (\% M/M) & $0.332^{*}$ & $0.354^{*}$ & 0.103 & 0.040 & 0.076 & $0.138^{\ddagger}$ & $0.258^{*}$ \\
Estradiol (E, pmol/l) & 0.003 & 0.004 & -0.014 & -0.005 & 0.089 & $0.201^{*}$ & $0.188^{*}$ \\
E $_{2} /$ SHBG ratio (ESR, pM/nM) & $0.274^{*}$ & $0.175^{\dagger}$ & 0.100 & -0.010 & 0.071 & $0.162^{\dagger}$ & $0.168^{\dagger}$ \\
\hline
\end{tabular}

SHBG, sex-hormone binding globulin; FAI, total testosterone/SHBG; cFT, calculated free testosterone; FTR, free testosterone/total testosterone ratio; LBM, total lean body mass; FBM, total fat body mass; MES, maximum extension strength; MGS, maximum handgrip strength; TUGT, timed up-and-go test; BMD, bone mineral density; L2-L4, lumbar spine; FN, femoral neck. ${ }^{\star} P<0.001 ;{ }^{\dagger} P<0.01 ;{ }^{\ddagger} P<0.05$; partial $r$, partial correlation.

and muscle mass and strength (13). Another study found that 46 postmenopausal women aged between 46 and 55 years old who used estrogens had decreased bioavailable testosterone and that this decrease was related to a decrease in LBM (24). In our population of 329 postmenopausal women, the age-related decreases in LBM, MES, and BMD in spine and FN were related to bioavailable testosterone (FAI, FTR) and estrogen (ESR).

\section{Changes in testosterone, estrogen, muscle mass, muscle strength, and BTM with age}

TT and total estrogen (TE) remained unchanged with ageing in our study. Cappola found a decrease in TT until the age of 80 years, but similar values in $80+$ compared with 65-68 years-old women, and no significant relation with the number of years since menopause (12). Risk factors for low testosterone have been documented in women aged 65 years and older and include bilateral oophorectomy, estrogen use, corticosteroid use, and low BMI (12). We found significant relations between bioavailable testosterone and estrogen and BMI.

Although, bioavailable estrogen (ESR) and testosterone (FTR) decreased with age, FAI, which is considered as a reliable index of free testosterone (17), did not change with age, as was also found by Cappola who measured free testosterone directly by dialysis (12).

As expected, we found significant loss of LBM, MES, MGS, and BMD in the spine and hip and an increase in TUGT with increasing age. The loss of MES $(-32 \%)$ and MGS $(-23 \%)$ between the ages of $<60$ years and $>75$ years was much more pronounced than the loss of LBM $(-5 \%)$, indicating also a decrease in muscle efficiency with ageing (25). Such losses of muscle mass and strength results in sarcopenia, which is a risk factor for falls and decreased quality of life (25).

Bone resorption (DPyr) increased with age, as has also been found by others (4). OC remained unchanged, while others found an increase in OC with age, but with unchanged bone alkaline phosphatase and carboxyterminal propeptide of type I procollagen (PICP) (4).

\section{Relations of testosterone and estrogen with muscle mass and muscle strength}

LBM and MES was significantly related to FAI and ESR. However, our age-adjusted analysis found FAI to be related to both LBM and MES, while ESR was only related to LBM. In addition, MES was independently related to LBM and FAI, but not to ESR. This indicates that muscle mass is testosterone and estrogen dependent, while muscle strength depends only on testosterone.

Although the effect of testosterone administration on muscle is variable, several intervention studies have reported that it leads to increased LBM, muscle size, and maximum voluntary strength in elderly men (10). The effect of testosterone and estrogen on muscle in postmenopausal women is still unclear (14). One confounder in the search for the relation between estrogen and muscle in intervention studies is the finding that estrogen replacement in postmenopausal women leads to decreased testosterone concentrations, and this decrease in testosterone is associated with a decreased LBM (14). By contrast, the combination of testosterone and estrogen leads to increased muscle mass (10).

Unlike MES, MGS was not related to any total or bioavailable testosterone or estrogen values. This is surprising, as MES, MGS, and LBM were highly intercorrelated. The decrease in muscle mass, strength, and power with ageing is caused by atrophy of muscle fibers, particularly those of type IIa. Testosterone binds to the androgen receptor in muscle tissue and stimulates protein synthesis in both type I (slow, aerobic) and type II (fast, anaerobic) muscle fibers. It is possible that the differences in the association of testosterone with MES and MGS are the result of a greater effect of testosterone in the most disused muscle, i.e., the quadriceps, in which the loss of maximum strength has been found to be $49 \%$ larger than the loss of MGS (26). Another possibility is that androgens mainly affect proximal limb muscles. Proximal muscle weakness is a typical clinical feature of Kennedy's disease, a genetic neurodegenerative disorder related to the androgen receptor, and in a 
knock-out animal model of this disease, mutant males exhibited androgen-dependent neuromuscular weakness (27). These findings suggest that measuring proximal limb muscle strength such as MES is mandatory in prospective studies of the effect of estrogen and testosterone on muscle strength.

No relation was found between testosterone and estrogen and TUGT, although the TUGT was significantly related to LBM and MES. The TUGT is, however, not only dependent on muscle mass and strength but also on coordination and balance. In a recent study, in elderly men testosterone supplementation did increase LBM but without any effect on the TUGT. Additional studies will be needed to analyze the relation between testosterone and estrogen and muscular functional repercussions.

\section{Relation of testosterone and estrogen with BMD}

The results of our study confirm the known relations between bone and circulating estrogen and testosterone in postmenopausal women (5). Circulating estrogen and testosterone levels are associated with $\operatorname{BMD}(3,4)$ and bone size at most skeletal locations (2). Circulating estrogen levels are also related to hip fracture risk and height loss (28), but not to vertebral fractures (29). We found that both the FAI and ESR were independently related to FN BMD, as has also been reported by others for postmenopausal women (4) and in elderly men (1). Prospective studies in elderly men indicate that testosterone is an independent risk factor for future fracture, but data for women are not available.

The BTM (OC and DPyr) were negatively related to ESR and FTR, but not to the FAI. This relation has also been found by others for estrogen and testosterone levels (4). These data confirm that low estrogen and testosterone are related to increased bone turnover in postmenopausal women, which is a risk factor for fractures $(30,31)$. The data suggest that the relation between BMD and estrogen and testosterone is in part mediated by the level of bone turnover. Indeed, we found OC to be negatively related to BMD in the spine and FN.

The pluripotent effects of testosterone and estrogen on bone and muscle open up new avenues for further research into interventions to increase muscle mass and strength. One option would be the development of selective androgen receptor modulators, some of which have been shown to have potent anabolic effects on bone and muscle $(10,32)$.

This study was subjected to some limitations. First, it was a cross-sectional population-based study, and longitudinal studies will be necessary to confirm our conclusions. Secondly, the women in this study were aged between 55 and 85 years. No data were available on women aged 85 years and over. Thirdly, no measured values of free testosterone and free estrogen were available, but only free testosterone calculated and free estrogen. Fourthly, 25-hydroxyvitamin D $(25(\mathrm{OH}) \mathrm{D})$ was not measured. There are studies that show that women with a hip fracture history, with extremely low 25(OH)D levels had reduced lower extremity muscle function (33) and another study showed that higher $1.25(\mathrm{OH})_{2} \mathrm{D}_{3}$ concentrations are associated with a lower fall risk in older women (34).

We conclude that in postmenopausal women, agerelated loss of LBM, MES (but not MGS), and BMD is partly dependent on the presence of endogenous bioavailable testosterone and estrogen. In addition to the well-known effects on bone, bioavailable testosterone and estrogen seem to play a role in the loss of lean mass and muscle strength that occur after menopause, although the cross-sectional nature of the study precludes a definitive conclusion.

\section{Declaration of interest}

There is no conflict of interest that could be perceived as prejudicing the impartiality of the research reported.

\section{Funding}

This project was supported by grants from Maastricht University. This research did not receive any specific grant from any funding agency in the public, commercial or not-for-profit sector.

\section{Author contribution statement}

TACMG is the principle investigator and the main author of the manuscript. PPG and GJD are the supervisors of the principle investigator and are, as well as JPJES, responsible for the medical and scientific content of the manuscript. BW is responsible for the statistical analyses of the data. All authors have read and approved the manuscript and agreed with publication of their names.

\section{Acknowledgements}

We would like to express appreciation to Mrs Pauline Versteeg, research assistant, who contributed substantially to the data acquisition.

\section{References}

1 Beld van den AW, Jong de FH, Grobbee DE, Pols HA \& Lamberts SW. Measures of bioavailable serum testosterone and estradiol and their relationships with muscle strength, bone density, and body composition in elderly men. Journal of Clinical Endocrinology and Metabolism 200085 3276-3282.

2 Khosla S \& Riggs BL. Pathophysiology of age-related bone loss and osteoporosis. Endocrinology and Metabolism Clinics of North America 200534 1015-1030 (xi).

3 Greendale GA, Edelstein S \& Barrett Connor E. Endogenous sex steroids and bone mineral density in older women and men: the Rancho Bernardo Study. Journal of Bone and Mineral Research 1997 12 1833-1843.

4 Khosla S, Melton LJ III, Atkinson EJ, O'Fallon WM, Klee GG \& Riggs BL. Relationship of serum sex steroid levels and bone turnover markers with bone mineral density in men and women: a key role for bioavailable estrogen. Journal of Clinical Endocrinology and Metabolism $1998 \mathbf{8 3} 2266-2274$. 
5 Vanderschueren D, Vandenput L, Boonen S, Lindberg MK, Bouillon R \& Ohlsson C. Androgens and bone. Endocrine Reviews 200425 389-425.

6 Lufkin EG, Wahner HW, O'Fallon WM, Hodgson SF, Kotowicz MA, Lane AW, Judd HL, Caplan RH \& Riggs BL. Treatment of postmenopausal osteoporosis with transdermal estrogen. Annals of Internal Medicine 1992117 1-9.

7 Snyder PJ, Peachey H, Hannoush P, Berlin JA, Loh L, Lenrow DA, Holmes JH, Dlewati A, Santanna J, Rosen CJ \& Strom BL. Effect of testosterone treatment on body composition and muscle strength in men over 65 years of age. Journal of Clinical Endocrinology and Metabolism $1999 \mathbf{8 4} 2647-2653$.

8 Herbst KL \& Bhasin S. Testosterone action on skeletal muscle. Current Opinion in Clinical Nutrition and Metabolic Care 20047 271-277.

9 Bhasin S, Calof OM, Storer TW, Lee ML, Mazer NA, Jasuja R, Montori VM, Gao W \& Dalton JT. Drug insight: testosterone and selective androgen receptor modulators as anabolic therapies for chronic illness and aging. Nature Clinical Practice. Endocrinology $\mathcal{E}$ Metabolism 20062 146-159.

10 Chen J, Kim J \& Dalton JT. Discovery and therapeutic promise of selective androgen receptor modulators. Molecular Interventions 20055 173-188.

11 Ferrando AA, Sheffield Moore M, Yeckel CW, Gilkison C, Jiang J, Achacosa A, Lieberman SA, Tipton K, Wolfe RR \& Urban RJ. Testosterone administration to older men improves muscle function: molecular and physiological mechanisms. American Journal of Physiology. Endocrinology and Metabolism 2002282 E601-E607.

12 Cappola AR, Ratcliffe SJ, Bhasin S, Blackman MR, Cauley J, Robbins J, Zmuda JM, Harris T \& Fried LP. Determinants of serum total and free testosterone levels in women over the age of 65 years. Journal of Clinical Endocrinology and Metabolism 2007 92 509-516.

13 Hakkinen K \& Pakarinen A. Muscle strength and serum testosterone, cortisol and SHBG concentrations in middle-aged and elderly men and women. Acta Physiologica Scandinavica 1993 148 199-207.

14 Padero MC, Bhasin S \& Friedman TC. Androgen supplementation in older women: too much hype, not enough data. Journal of the American Geriatrics Society 200250 1131-1140.

15 Sands R \& Studd J. Exogenous androgens in postmenopausal women. American Journal of Medicine 199598 76s-79s.

16 Pardridge WM. Serum bioavailability of sex steroid hormones. Clinics in Endocrinology and Metabolism 198615 259-278.

17 Vermeulen A, Verdonck L \& Kaufman JM. A critical evaluation of simple methods for the estimation of free testosterone in serum. Journal of Clinical Endocrinology and Metabolism $1999 \mathbf{8 4}$ 3666-3672.

18 Voort van der DJM, Geusens PP \& Dinant GJ. Risk factors for osteoporosis related to their outcome: fractures. Osteoporosis International $200112630-638$.

19 Podsiadlo D \& Richardson S. The timed 'Up \& Go': a test of basic functional mobility for frail elderly persons. Journal of the American Geriatrics Society 199139 142-148.

20 Andree G, Bachani B, Pregger K, Durham AP \& El Shami AS. Use of an extended NCCLS EP5 Protocol to characterize the precision and sensitivity of the IMMULITE 2000 total testosterone assay. Clinical Chemistry $2004 \mathbf{5 0}$ A100.
21 Field A. Discovering Statistics Using SPSS for Windows Trowbridge: The Cromwell Press Ltd, 2000.

22 Anonymous. Coat a Count - estradiol. Siemens Medical Solutions Diagnostics, 2006.

23 Anonymous. Coat a Count - total testosterone. Siemens Medical Solutions Diagnostics, 2006.

24 Gower BA \& Nyman L. Associations among oral estrogen use, free testosterone concentration, and lean body mass among postmenopausal women. Journal of Clinical Endocrinology and Metabolism 200085 4476-4480.

25 Morley JE, Baumgartner RN, Roubenoff R, Mayer J \& Nair KS. Sarcopenia. Journal of Laboratory and Clinical Medicine 2001137 231-243.

26 Lynch NA, Metter EJ, Lindle RS, Fozard JL, Tobin JD, Roy TA, Fleg JL \& Hurley BF. Muscle quality. I. Age-associated differences between arm and leg muscle groups. Journal of Applied Physiology 199986 188-194.

27 Yu Z, Dadgar N, Albertelli M, Gruis K, Jordan C, Robins DM \& Lieberman AP. Androgen-dependent pathology demonstrates myopathic contribution to the Kennedy disease phenotype in a mouse knock-in model. Journal of Clinical Investigation $2006 \mathbf{1 1 6}$ 2663-2672.

28 Cauley JA, Black DM, Barrett Connor E, Harris F, Shields K, Applegate W \& Cummings SR. Effects of hormone replacement therapy on clinical fractures and height loss: the Heart and Estrogen/Progestin Replacement Study (HERS). American Journal of Medicine $2001110442-450$.

29 Nevitt MC, Cummings SR, Stone KL, Palermo L, Black DM, Bauer DC, Genant HK, Hochberg MC, Ensrud KE, Hillier TA \& Cauley JA. Risk factors for a first-incident radiographic vertebral fracture in women $>$ or $=65$ years of age: the study of osteoporotic fractures. Journal of Bone and Mineral Research 2005 20 131-140.

30 Garnero P \& Delmas PD. Bone markers. Baillière's Clinical Rheumatology 199711 517-537.

31 Garnero P, Hausherr E, Chapuy MC, Marcelli C, Grandjean H, Muller C, Cormier C, Breart G, Meunier PJ \& Delmas PD. Markers of bone resorption predict hip fracture in elderly women: the EPIDOS Prospective Study. Journal of Bone and Mineral Research 199611 1531-1538.

32 Wilson EM. Muscle-bound? A tissue-selective nonsteroidal androgen receptor modulator Endocrinology 2007 148 1-3.

33 LeBoff MS, Hawkes WG, Glowacki J, Yu Yahiro J, Hurwitz S \& Magaziner J. Vitamin D-deficiency and post-fracture changes in lower extremity function and falls in women with hip fractures. Osteoporosis International 200819 1283-1290.

34 Faulkner KA, Cauley JA, Zmuda JM, Landsittel DP, Newman AB, Studenski SA, Redfern MS, Ensrud KE, Fink HA, Lane NE \& Nevitt MC. Higher 1,25-dihydroxyvitamin D3 concentrations associated with lower fall rates in older community-dwelling women. Osteoporosis International 200617 1318-1328.

Received 1 December 2008

Accepted 26 January 2009 